\title{
Comment to: the use of silicone oil-RMN3 (Oxane HD) as heavier-than-water internal tamponade in complicated inferior retinal detachment surgery
}

\author{
Kamron Nasser Khan • Rubina Rahman
}

Received: 14 June 2010 / Accepted: 5 August 2010/Published online: 25 August 2010

(C) Springer-Verlag 2010

\section{Dear Sir,}

We read with interest the recent article of Doctors Whickham et al. on their use of Oxane in the treatment of complex inferior retinal detachment, and would like to question a few points and make our own comments.

When classifying the grade of retinal detachment in the study group, three recurrent detachments were said not to have grade C PVR, whilst in the overall grade of PVR in the same table two patients in the study group had non-grade $\mathrm{C}$ PVR (one with no PVR, and one grade B). The median duration of retinal detachment for the study group lies outside the inter-quartile range for that group and is probably at least double the duration of the control group in reality.

As the presence of residual silicone oil is stated as a secondary outcome, we feel a more detailed account of the technique of removal should be presented. Heavy silicone oil is acknowledged to be stickier and hence more difficult to remove than standard oil. We have modified our original technique after early experiences with Oxane and now prefer to perform repeated fluid-air exchanges unless all visible oil droplets are removed from the vitreous cavity.

The authors rightly state that in their small nonrandomised case series there is no statistical difference in primary outcome measures between the two groups. However, the two groups are not well-matched for comparison, as patients treated with conventional oil were asked to posture for 5 days postoperatively, while the HSO group did not posture. This difference in posturing instruction alone could have resulted in improved retinal reattachment rates with standard oil.

An alternative conclusion that their results would support is that for patients who are unable or unwilling to posture after complex inferior retinal detachment surgery the use of Oxane should be considered.

The authors have reported hypotony in four cases, one in the standard and three in the HSO group. It is not clear from the data how many patients underwent inferior relaxing retinotomies, as this may have contributed to hypotony by improving uveo-scleral outflow.

Oil was said to be removed at 3 months (90 days) unless contraindicated. Where this data is presented (Table 5), the mean (and median) ROSO time is 5.3 months (160 days) which is significantly longer, and if earlier oil removal were possible it would be interesting to compare the complication profile. Our personal experience with HSO (Oxane HD) in the context of failed macular hole repair (unpublished data, manuscript in preparation) indicates that removal as early as 6 weeks after insertion significantly lowers the incidence of postoperative uveitis and glaucoma.

Kamron Khan

Rubina Rahman
K. N. Khan $(\varangle) \cdot$ R. Rahman

Calderdale Royal Hospital,

Salterhebble, Halifax, West Yorkshire HX3 0PW, UK

e-mail:kamronkhan@yahoo.com 